\title{
Design and reliability analysis of image type head gesture interface in distributed control system
}

\author{
Chern-Sheng Lin ${ }^{1 *}$, Hsiang-Wen Tseng ${ }^{1}$, Yun-Long Lay ${ }^{2}$, Chia-Tse Chen ${ }^{1}$ and Mau-Shiun Yeh ${ }^{3}$ \\ ${ }_{1 *}^{*}$ Department of Automatic Control Engineering, Feng Chia University, Taichung, Taiwan \\ ${ }^{2}$ Department of Electronic Engineering, National Chin-Yi University of Technology, Taichung, Taiwan \\ ${ }^{3}$ Chung-Shan Institute of Science \& Technology, Lung-Tan, Tao-Yuan, Taiwan \\ lincs@fcu.edu.tw
}

\begin{abstract}
In this study, a novel method for head-controlled human-machine interface system is presented. The proposed system is composed of the image type head gesture interface and the distributed control system (DCS). In the head gesture interface, the frame grabber and CCD camera track the lighting pointer of double-source eyeglass frame. The computer programs with new algorithms locate the center-point of the head, transferring it to the screen coordinates, and then the user can control the cursor by head motions. The DCS with controlling commands and could be used for driving the relative hardware to assist the system more stably. The failure rate was obtained by testing in 60 days. The present system can provide an economical and effective method for reliability. It is also useful in the field of interface and technology.
\end{abstract}

Keywords: Head gesture interface, Distributed control system, Petri net architecture analysis

\section{Introduction}

The research method of this study is focused on the functional improvement of the image type head gesture interface system (Betke et al, 2002; Lin et al, 2006a), as well as the mechanical-electric integration and application of DCS. The movement of head gesture was detected by using a hardware device which changes the position and quantity of light source, thus forming a highly efficient man-machine interface. This man-machine interface mainly used a head controlled light source, instead of a mouse, to drive the cursor on the screen; thus, the operators can switch on computers and work by just moving their heads.

\section{Fig. 1. Architecture of double light source} video head gesture control

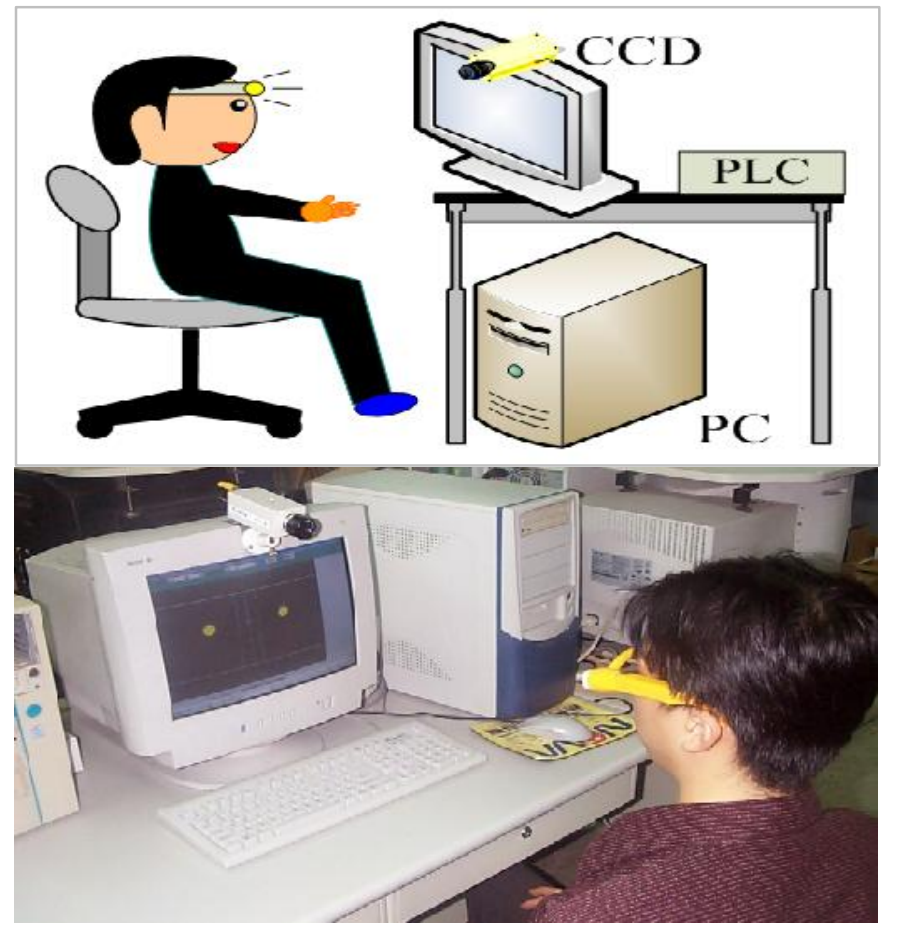

The head gesture interface system uses a CCD camera and an image capture card to capture the bright spot of head-mounted double light source, and converts the digital video signal into coordinates of cursor movement on the computer screen by using image processing technology (Lin, 2002; Lin et al, 2004; Lin et al, 2006b; Barr et al, 2007; Ju, 2009). Its architecture is shown in Fig. 1. In the double light source video head gesture interface system, the light source is placed on the user's head. The light source is as an emitter, and a CCD camera is mounted above the screen. The CCD camera is used for detecting the position of light source, and the cursor movement is controlled by the movement of the head through the image capture card.

In this study, the DCS control system is a computeraided tool developed mainly for patients with motor neuron disease (MND) or persons with physical and mental disability. Since the controlled object can be a household appliance or a mechanical arm, the system was designed for easy operation, simple construction and economy. It adopted a PLC as the input/output module, and used the computer to generate instruction ladder diagram by using FXGP-WIN-T software. It then used a new method to design the monitor menu of humanmachine interface, and set the communication protocol. The computer communicated with PLC through the RS232 interface and RS422 adapter, and transmitted the instruction to PLC, in order to control the output of PLC, and achieve on-line monitoring.

The distributed Control System (DCS) composes several directly linked control systems in a single independent control unit based on the demand of system control function, and can share data with other indirectly linked control systems through the communication network (Chen, 2001; Lin et al, 2008). It is an open integration, and it is distributed and specially designed for industrial auto-control. It is mainly composed of a computer, PLC, motor controller and communication network, including control level 3 to level 0 (Level 3 Level 
Fig. 2. Image of bright spot in head control system
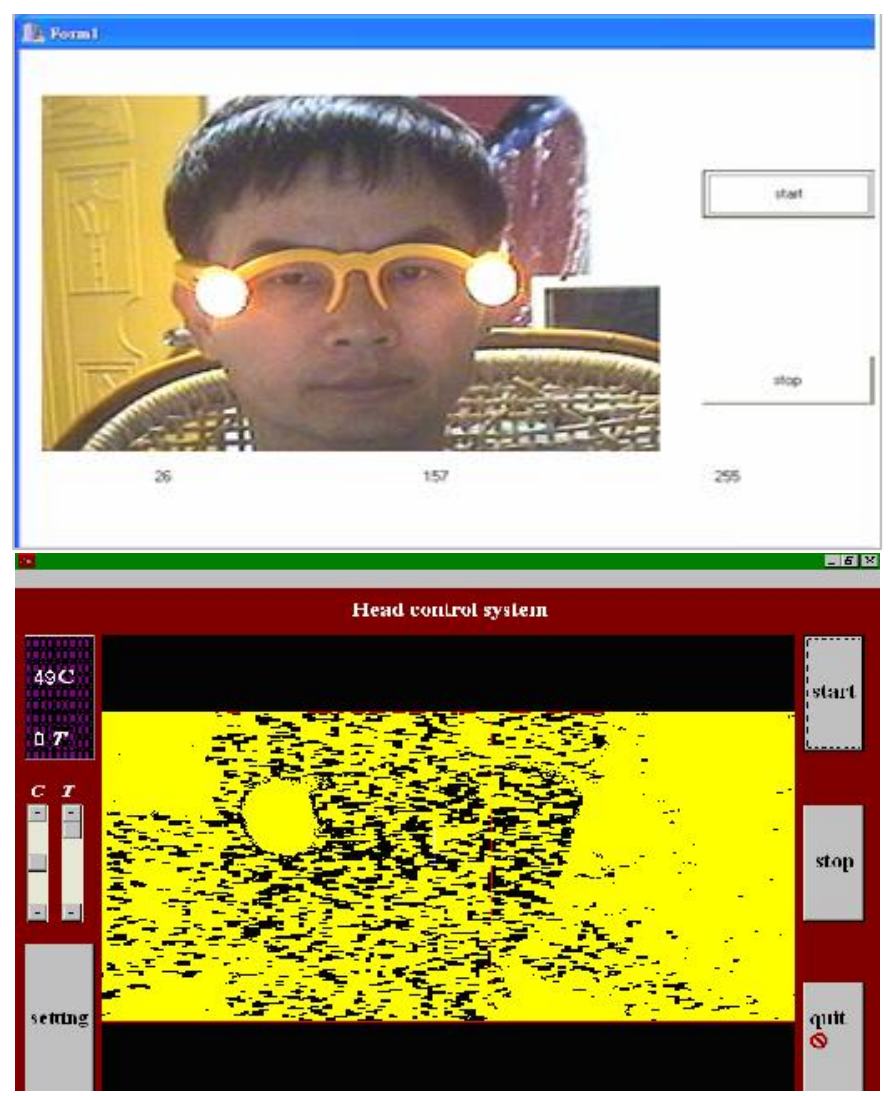

Fig.3. Regional coordinates of bright spot.

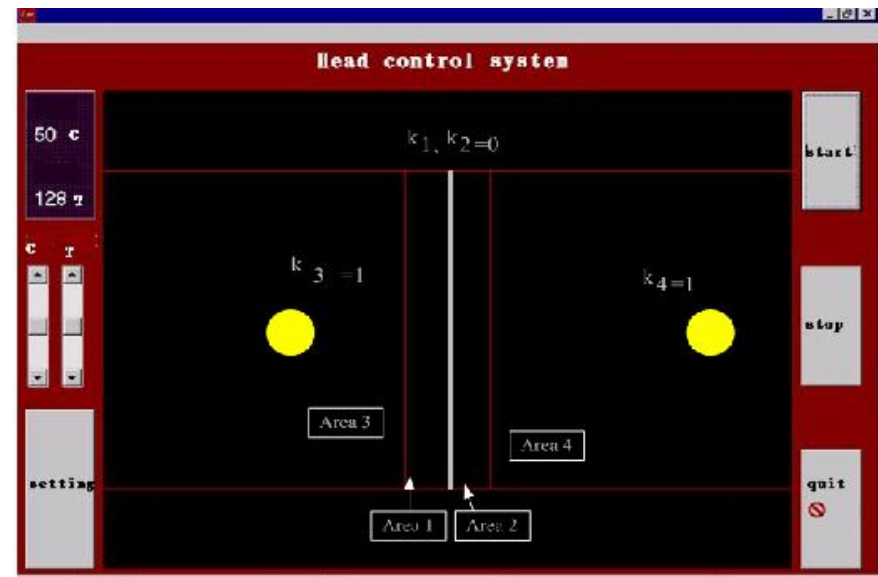

0 ), connected by the network. The DCS control system uses the man-machine interface (SCADA or HMI) to set all machine systems directly, and uses the screen to monitor relevant configurations and structures. The system can set the state demand of every machine, such as electric current, speed, temperature, pressure, bound, and default. It processes relevant numerical values via PLC first, and after calculating the entire data set, they are transferred to the motor controller or linked control unit via communication network, in order to control the motor speed and other control equipments (Lee, 1999; Allison et al, 1996; Lin et al, 2009a). When the system Research article "Head gesture interface" COndian Society for Education and Environment (iSee) response has diverged from its specification, the system can assume the parameters are independent and identically distributed among fault observations with the machine learning techniques and Petri Net architecture analysis (Peraira et al, 2011).

Method and analysis of head gesture interface system Capture the bright spot of image

When the images of light source movement are captured by CCD camera driven by the image capture card, the image data are transmitted to the computer and stored in a fixed address. Therefore, each pixel in the image is composed of three groups of Red, Green and Blue images within $0 \sim 255$ brightness.

The traditional image processing mode has low speed, thus is time-consuming. This study specified a search boundary. Fig. 2 is the image of the bright spots captured by the system. The rectangle region outside the bright spot is the image search boundary, which can shorten the image processing time, as well as avoid noise which may cause misjudgment of the program (Yoon et al, 2001; Lin et al, 2007; Lin et al, 2009b).

Binary operation was carried out for the search area of images with gray level $f(i, j)$. Basically, it is for separating bright spots from the images, allows the system to lock the correct movement of the bright spot. The binary image $g(i, j)$ is defined as

$$
g(i, j)=\left\{\begin{array}{l}
255, f(i, j)>T \\
0, f(i, j) \leq T
\end{array}\right.
$$

where, $T$ is the binary threshold value.

\section{Calculate central coordinates}

During the binarization of image, the horizontal coordinate $i$ values and longitudinal coordinate $j$ values of pixels are added together, which have gray-scale value greater than the threshold value $T$. If $k$ pixels greater than the threshold value $T$ within the search area, the central coordinates of bright spot could be obtained based on the following equation.

$$
(m, n)=\frac{1}{k}\left[\sum_{1}^{k} i, \sum_{1}^{k} j\right]
$$

In Fig. $3, k_{1}, k_{2}, k_{3}$ and $k_{4}$ are the number of bright
Fig. 4. Coordinates of the light source

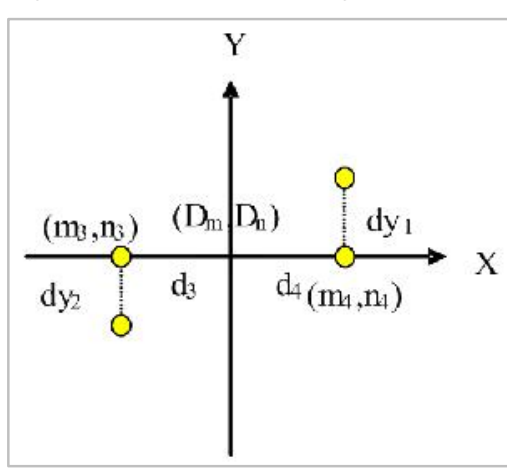
spots searching four areas, and $m_{I}$ represents the $\mathrm{X}$-axis coordinates of light source center entered this area I; $n_{1}$ represents the $\mathrm{Y}$-axis coordinates of light source center entered area I; $\mathrm{dy}_{1}$ is the distance from right light source to window center on y-axis, dy2 is the distance from left light 
source to the center on the $y$-axis; d3 is the distance from the left light source to the center on the $x$-axis, and $d 4$ is the distance from right light source to center on $x$-axis.

At the beginning of the program, $k_{3}>0, k_{4}>0, k_{1}=0$, and $k_{2}=0$ can be known, and the double light source center coordinates $\left(D_{m}, D_{n}\right)$ are given by (Fig. 4)

$$
\begin{aligned}
& D_{m}=\frac{m_{3}+m_{4}}{2}, \quad D_{n}=\frac{n_{3}+n_{4}}{2} \\
& d_{3}=\left(\frac{m_{3}+m_{4}}{2}\right)-m_{3}, \quad d_{4}=m_{4}-\left(\frac{m_{3}+m_{4}}{2}\right) \\
& \text { (4) } \\
& d y_{1}=\left[\frac{n_{3}+n_{4}}{2}\right]-n_{4}, d y_{2}=n_{3}-\left[\frac{n_{3}+n_{4}}{2}\right]
\end{aligned}
$$

We can divide six operation conditions to three case: (a) Head mounted light source shifts to the right - Case 1, (b) Head mounted light source shifts to the right - Case 2, (c)Head mounted light source shifts to the right - Case 3, (d)Head mounted light source shifts to the left - Case 1, (e)Head mounted light source shifts to the left - Case 2, (f)Head mounted light source shifts to the left - Case 3.

When the spot moves left, the corresponding central coordinates $(\mathrm{Dm}, \mathrm{Dn})$ are related by

(1) As shown in Fig. 5(a), if $k_{3}=0$ (user is close to CCD) or $\mathrm{k}_{2}>0, \mathrm{k}_{4}>0$ (user is far from CCD), then

$\mathrm{D}_{\mathrm{m}}=\mathrm{m}_{4}-\mathrm{d}_{4}$ and $\mathrm{D}_{\mathrm{n}}=\mathrm{n}_{4}+\mathrm{dy} \mathrm{y}_{1}$

(2) As shown in Fig. 5(b), if $k_{2}>0, k_{4}=0$ (user is far from CCD), then $m=m_{2}+\left(d_{i a m \times 2} / 2\right)$,

$D_{m}=m-d_{4}, D_{n}=n_{2}+d y_{1}$ and $D_{n}=n_{2} \quad d_{\text {iamx2 }}$ is $k_{2}$ spot diameter

(3) As shown in Fig. 5(c), if $k_{1}>0, k_{2}=0$ (user is far from $C C D)$, then $m=m_{1}+\left(d_{\text {iamx } 1} / 2\right)$

$D_{m}=m-d_{4}$ and $D_{n}=n_{1}+d y_{1} \quad d_{i a m \times 1}$ is $k_{1}$ spot diameter

When spot moves toward right, the corresponding central coordinates (Dm, Dn) are given by

(1) As shown in Fig. 5(d), if $k_{4}=0$ (user is close to CCD) or $k_{1}>0, k_{3}>0$ (user is far from CCD),

then

$D_{m}=m_{3}+d_{3}$ and $D_{n}=n_{3}-d y_{2}$

(2) As shown in Fig. $5(\mathrm{e})$, if $\mathrm{k}_{1}>0, \mathrm{k}_{3}=0$ (user is far from CCD), then $m=m_{1}+\left(d_{i a m \times 1} / 2\right)$

$D_{m}=m+d_{3}$ and $D_{n}=n_{1}-d y_{2}$

(3) As shown in Fig. 5(f), if $k_{2}>0, k_{1}=0$ (user is far from CCD), then $m=m_{2}-\left(d_{\text {diam } x 2} / 2\right)$,

$\mathrm{D}_{\mathrm{m}}=\mathrm{m}+\mathrm{d}_{3}$ and $\mathrm{D}_{\mathrm{n}}=\mathrm{n}_{2}-\mathrm{dy} \mathrm{y}_{2}$

Integration with PLC monitoring system

The architecture of PLC monitoring system can be divided into PC and PLC. The desktop computer is the main control center, and PLC is the minor control center. When user starts up the head gesture interface system, the program enters the monitoring system menu, and users can use the head-mounted double light source to select the control button. Then PC transmits the command to PLC via RS232, PLC uses PC signal to then the connection point of the output relay controls the action of household electric appliances (Fig. 6).

In the connection of PLC to PC, the hardware wiring needs to be handled first. PLC has RS422 COM port, and RS422 can be converted to RS232 interface by inserting an AX-232AW module, thus, it can be connected to the communication port of PC.

Petri Net (Fig. 7) provides a dynamic analysis of graphs (Lin and choiu,1997). It is a directional and binary graphic architecture, and is composed of two base elements, which are in Place (indicated by $\mathrm{P}$ ) and Transition (indicated by $\mathrm{t}$ ). They are connected by an arrow headed straight line or Arc to indicate the traveling path and direction of Place or Transition. In this study, the architecture of Petri Net is indicated by $C$, if $C=(P, T, I$, $O)$. The definition is as follows:

$P=\{p 1, p 2, \ldots . p n\}, T=\{t 1, t 2, \ldots t m\}$

$\mathrm{I}:(P \times T) \rightarrow C, I$ is the input state matrix reflected on $T$ by $P$ by $T$

If $A=O-I$, it is called Incidence Matrix, and the following equation can be deduced:

$$
M_{d}=M_{0}+A \sum_{i=1}^{d} U_{i}
$$

Based on the above mathematical model and simulation of computer programming, its dynamic behavior can be presented. This study designed the sequence control program of PLC based on the foundation of Petri Net. With this efficient mode, the program designing process can be simplified. Thus, the programming time can be shortened and human errors can be avoided, thus increasing the efficiency of control programming. When the dynamic simulation matches the expected requirement, practical monitoring operation can be carried out, so that the testing time after the completion of program can be saved.

\section{Analysis of reliability}

Most testers in the head gesture interface system are young graduate students, who are active and have quick responses, and make fewer human errors (Huang et al, 2003). However, the main users of this system are patients with MND and severely disabled persons, who have difficulties in moving and need care of their families and nurses. If $X$ represents an event, then its probability is given by $P(X)$

$$
P(X)=\lim _{N \rightarrow \infty} \frac{n}{N}, 0 \mathrm{P}(\mathrm{X}) 1
$$

$\mathrm{N}$ : Total amount of tests $\mathrm{n}$ : Frequency of event $\mathrm{X}$ during $\mathrm{N}$ tests

If $X_{1}, X_{2}, \ldots ., X n$ are independent to each other,

$\mathrm{P}\left(\mathrm{X}_{1} \mathrm{X}_{2} \quad \ldots . \mathrm{Xn}\right)=\prod_{i=1}^{n} P\left(X_{i}\right)$. control the output relay based on built-in program, and $\mathrm{O}:(\mathrm{P} \times \mathrm{T}) \rightarrow \mathrm{C}, \mathrm{O}$ is the output state matrix reflected on $\mathrm{P}$ 


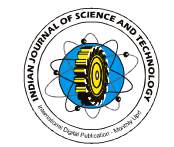

Fig. 5. Six operation conditions: (a), (b), and (c) shows the cases of head mounted light source shifts to the right and (d), (e), and (f) shows the cases of head mounted light source shifts to the left

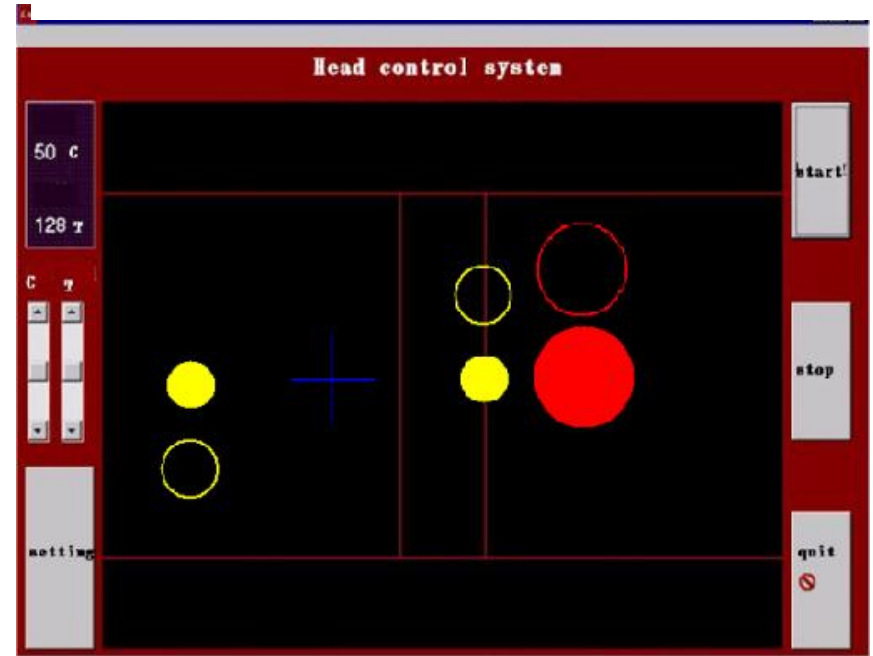

(a)

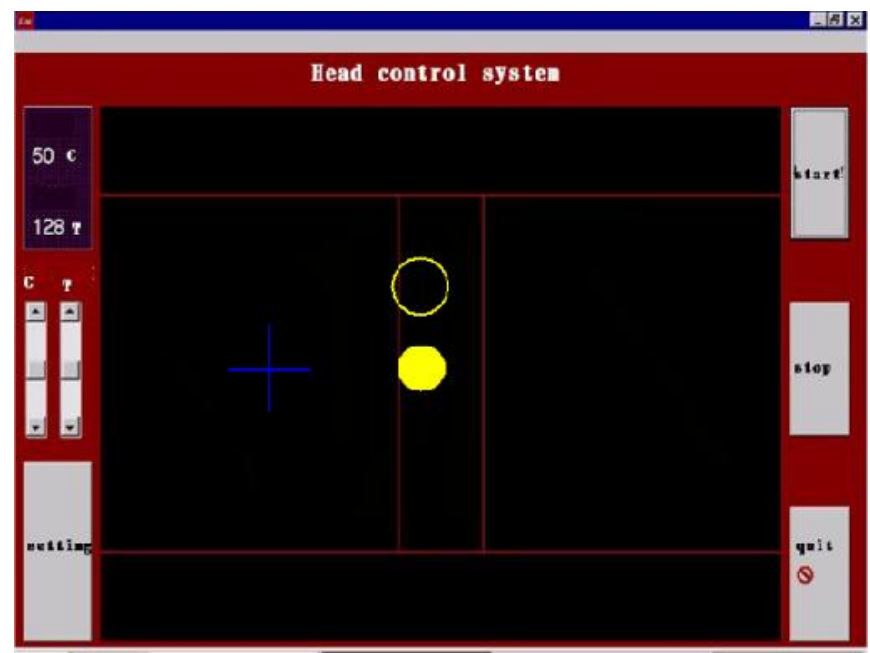

(c)

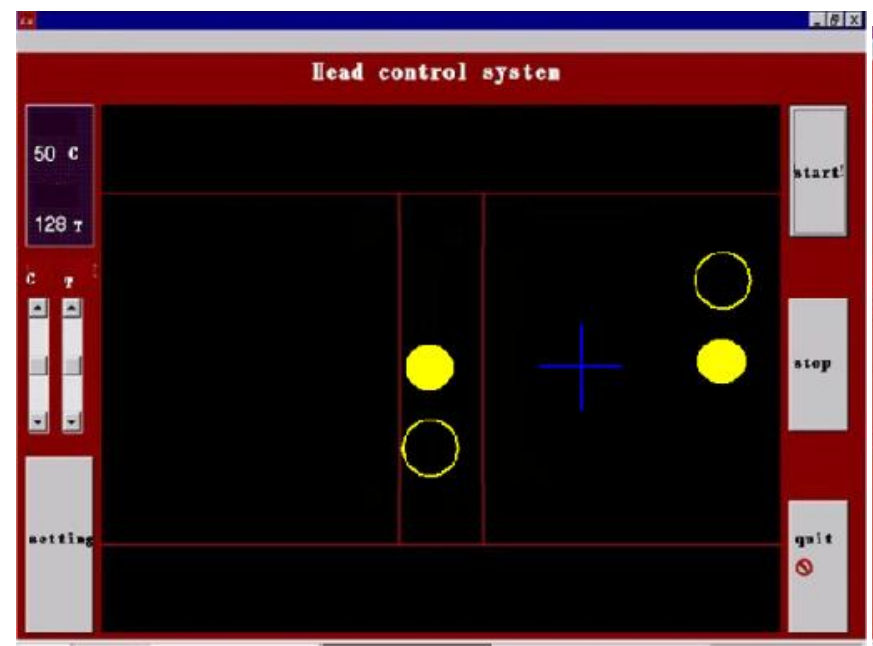

(e)

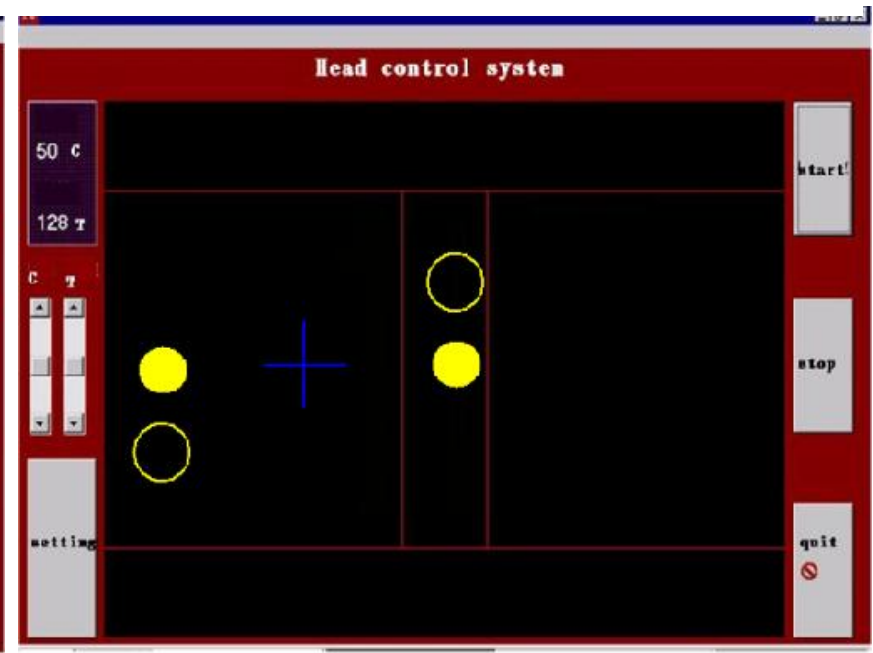

(b)

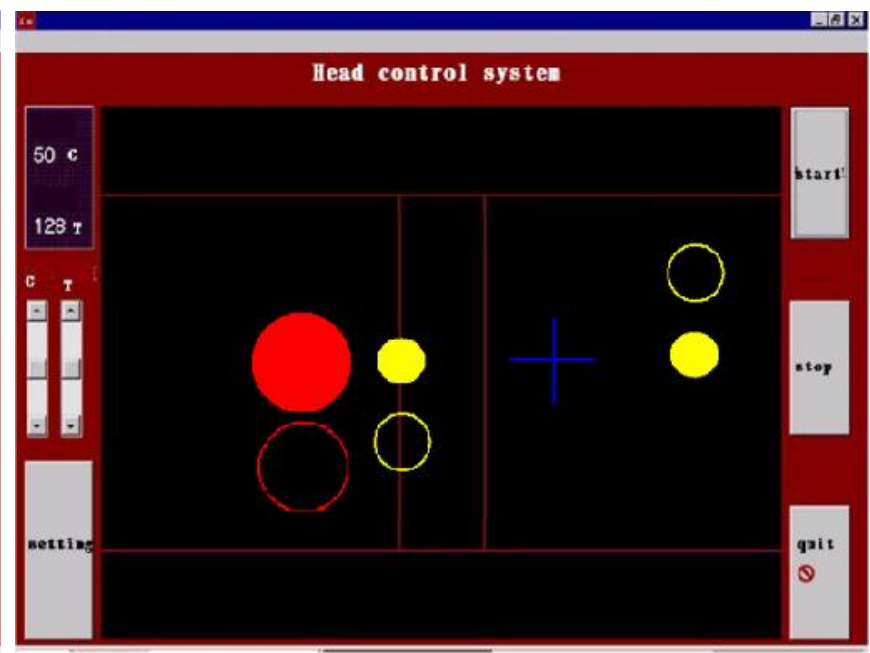

(d)

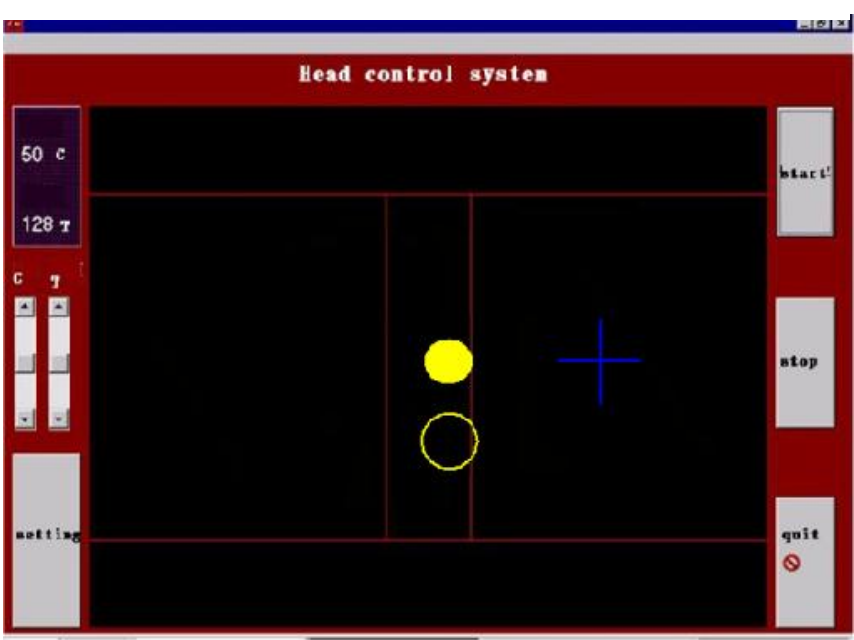

(f) 
Fig. 6. System architecture

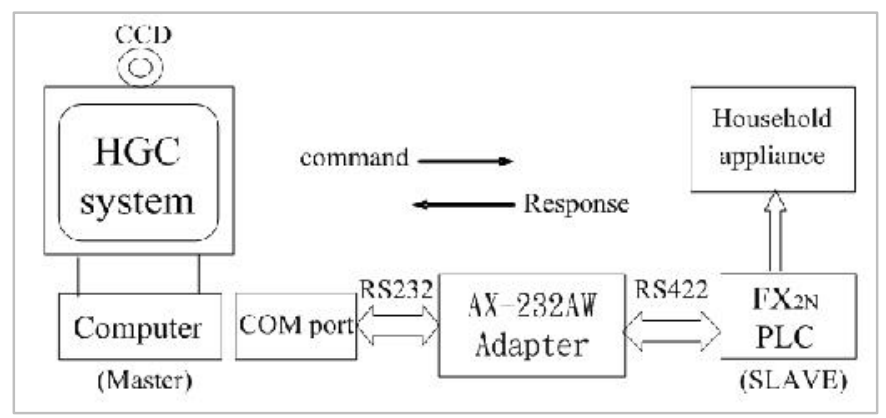

Fig. 7. (a) Unmarked petri net and (b) Marked petri net

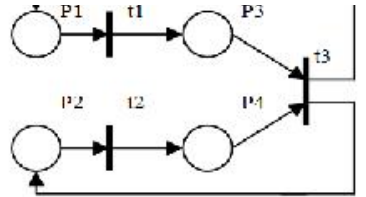

(a)

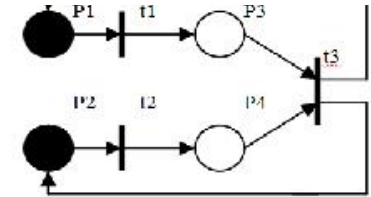

(b)
Representing the probability that event $X_{1}, X_{2}, \ldots ., X n$ occur at the same time

$\mathrm{P}\left(\mathrm{X}_{1} \mathrm{X}_{2} \quad \ldots . \mathrm{Xn}\right)=1-\prod_{i=1}^{n}\left[1-P\left(X_{i}\right)\right]$

Representing the probability that any event of $X_{1}, X_{2}, \ldots$. , $X n$ occurs. If $R_{n}$ is the probability that the system still functions after $n$ demands (i.e. reliability), and $X_{n}$ means event $X$ becomes successful event after $n$ demands, then $R_{n}=P\left(X_{1}\right) P\left(X_{2}\right) \ldots . P\left(X_{n}\right)$

If $\mathrm{P}\left(\mathrm{X}_{1}\right)=\mathrm{P}\left(\mathrm{X}_{2}\right)=\ldots .=\mathrm{P}\left(\mathrm{X}_{n}\right)=\mathrm{q}$, i.e. $P\left(\bar{X}_{n}\right)=1-q=p$

(failure probability), then

$\mathrm{R}_{\mathrm{n}}=\mathrm{q}^{\mathrm{n}}=(1-\mathrm{p})^{\mathrm{n}} \Rightarrow \ln \mathrm{R}_{\mathrm{n}}=\mathrm{n} \ln (1-\mathrm{p})$

If $p<<1$, then $\ln (1-p)-p$, In $R_{n}=-n p \Rightarrow R_{n}=e^{-n p}$

According to Eq.(16), the reliability $\mathrm{Rn}$ shows an exponential decline with the demand times $\mathrm{n}$ (Lin et al, 2012). If $f(t)$ is the failure probability density function pdf, then the sum of failure probability

$$
F(t)=\int_{0}^{t} f(t) d t
$$

Reliability $R(t)=\int_{t}^{\infty} f(t) d t$

Failure rate $\lambda(t)=\frac{f(t)}{R(t)}$

Mean time to failure

$$
M T T F=\frac{1}{\lambda}=\int_{0}^{\infty} R(t) d t
$$

Describe TTF by Weibull distribution

Failure rate $\lambda(t)=\frac{\beta}{\alpha}\left(\frac{t}{\alpha}\right)^{\beta-1}$

Reliability

$$
R(t)=\exp \left[-\int_{0}^{t} \lambda(t) d t\right]=\exp \left[-\left(\frac{t}{\alpha}\right)^{\beta}\right]
$$

$\alpha, \beta$ : Time variation parameters

$\beta=1$ can describe the fixed failure rate of random failure

\begin{tabular}{|c|c|c|c|c|c|c|c|}
\hline \multicolumn{8}{|c|}{ Times of test (j) } \\
\hline $\mathrm{t} 1$ & $y 1,1$ & $\mathrm{Y} 2,1$ & $\mathrm{Y} 3,1$ & $\ldots$ & $\ldots$ & Yn,1 & \multirow{5}{*}{ 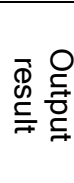 } \\
\hline $\mathrm{T} 2$ & $y 1,2$ & $\mathrm{Y} 2,2$ & $\mathrm{Y} 3,2$ & $\ldots$ & $\ldots$ & Yn,2 & \\
\hline T3 & $y 1,3$ & Y2,3 & Y3,3 & , & ... & Yn,3 & \\
\hline$\ldots$ & $\ldots$ & ... & $\ldots$ & $\ldots$ & $\ldots$ & $\ldots$ & \\
\hline $\mathrm{tm}$ & $\mathrm{y} 1, \mathrm{~m}$ & $\mathrm{Y} 2, \mathrm{~m}$ & $\mathrm{Y} 3, \mathrm{~m}$ & $\ldots$ & $\ldots$ & $\mathrm{Yn}, \mathrm{m}$ & \\
\hline
\end{tabular}
$\beta>1$ can describe the effect of increasing failure rate

Table 1. Test data matrix

$\beta<1$ can describe the effect of decreasing failure rate During each observation time $t_{j}(j=1,2, \ldots, m), n$ tests were conducted, and the output result $Y(t)$ is shown in Table 1. Based on the above matrix, $p d f=f_{Y}(y ; t)$ is obtained, when a failure event $D$ occurs, substitute it into Eq.(18) to obtain the reliability $R(\mathrm{t})=\mathrm{P}\{\mathrm{Y}(\mathrm{t})>\mathrm{D}\}=\int_{D}^{\infty} f_{Y}(y ; t) d t$

$$
\begin{aligned}
& \mathrm{R}(0)=1, \mathrm{R}\left({ }^{\infty}\right)=0, \mathrm{R}(\mathrm{t})=1-\mathrm{F}_{\mathrm{Y}}(\mathrm{y} ; \mathrm{t})=1-\int_{0}^{D} f_{Y}(y ; t) d t \\
& \mathrm{f}_{\mathrm{Y}}(\mathrm{y} ; \mathrm{t})=\square \frac{d}{d t} R(t)
\end{aligned}
$$

And failure rate $\lambda(t)=-\frac{1}{R(t)} \frac{d R(t)}{d t}$

$M T T F=\int_{0}^{\infty} R(t) d t$

\section{Experimental results}

If the head gesture control monitoring system is tested everyday, select the button for 10 times, and record the test results of two months, as shown in Table 2. The output result 1 means normal output, and 0 means abnormal output, including system failure resulted from failures caused by human factors or hardware and software.

1. Figure out the probability that events with abnormal output happen based on total times

$$
P(X)=\lim _{N \rightarrow 600} \frac{n}{N}=\frac{2}{600} \cong 0.003 .
$$

\begin{tabular}{|c|c|c|c|c|c|c|c|c|c|c|c|}
\hline \multicolumn{12}{|c|}{ Times of test (j) } \\
\hline Time(Days) & 10 & 20 & 30 & 40 & 50 & 60 & 70 & 80 & 90 & 100 & \multirow{7}{*}{$\stackrel{\mathscr{D}}{\rightleftharpoons}$} \\
\hline 10 & 1 & 1 & 1 & 1 & 1 & 1 & 1 & 1 & 1 & 1 & \\
\hline 20 & 1 & 1 & 1 & 1 & 1 & 1 & 1 & 1 & 1 & 1 & \\
\hline 30 & 1 & 1 & 0 & 1 & 1 & 1 & 1 & 1 & 1 & 1 & \\
\hline 40 & 1 & 1 & 1 & 1 & 1 & 1 & 1 & 1 & 1 & 1 & \\
\hline 50 & 1 & 1 & 1 & 1 & 1 & 1 & 0 & 1 & 1 & 1 & \\
\hline 60 & 1 & 1 & 1 & 1 & 1 & 1 & 1 & 1 & 1 & 1 & \\
\hline
\end{tabular}

2. Reliability $R \quad{ }_{(230+470)}=(1 \square \mathrm{p})^{\mathrm{n}}=\mathrm{q}^{\mathrm{n}}=(1-0.003)^{2}=99.4 \%$, failure probability $\mathrm{p}=0.003$.

3. If the failure rate is obtained by testing the total Table 2. Test output matrix by HGC 
number of days $\lambda=2 / 60=0.03$ times/day.

4. $\mathrm{F}(60)=\mathrm{P}(\mathrm{T} \quad 60)=\int_{0}^{60} f(t) d t=e^{-0.03 \times 60}=0.165$

5. $\mathrm{R}(60)=1 \square \mathrm{F}(\mathrm{t})=1 \square 0.165=0.835=83.5 \%$

6. $M T T F=\int_{0}^{\infty} R(t) d t=\frac{1}{\lambda}=33$ (days)

In fact, the failure rate of system is not distributed equally; it increases gradually after a period of time, so Weibull distribution is used to describe its TTF.

According to Table 2, when $\alpha$ is 60 days, $\beta$ is 2

(2 times of failure), the mean life time of PC is $2 \sim 5$ years, so $\alpha$ is set as 1825 days, and $\beta$ as $2, t$ as 730 .

7. Two-year failure rate

$$
\lambda(t)=\frac{\beta}{\alpha}\left(\frac{t}{\alpha}\right)^{\beta-1}=\frac{2}{1825}\left(\frac{730}{1825}\right)^{2-1} \cong 0.00044
$$

8. Design lifetime

$$
R(t)=\exp \left[-\left(\frac{t}{\alpha}\right)^{\beta}\right] \Rightarrow t=\alpha\left\{\ln \left[\frac{1}{R(t)}\right]\right\}^{\frac{1}{\beta}}=1825\left\{\ln \left[\frac{1}{0.835}\right]\right\}^{\frac{1}{2}}=775
$$

days

According to the analysis of Weibull distribution, after two years of operation, the failure rate of system increases, and the failure probability becomes higher.

Failure refers to a state, in which the head gesture interface system cannot exert the preset function, including: (1) Error can be classified as human error and system error. One of the reasons of human error is its inherent weakness failure. Since patients with MND and severely disabled patients cannot shake their heads flexibly, they may cause error events when using the head gesture interface system. While system errors exist in $P C$ and PLC, or between $C C D$ and PC, when the adjustable camera (CCD) above the PC is fixed at an improper position or the focus is adjusted improperly, or the parameter settings of PC image capturing menu (Fig. 8(a)) are incorrect, such as the brightness, contrast, and threshold. All of these may cause error events during system operation. Fig. 8 (b) is the abnormal parameter setting, which may cause malfunction. The coefficient of relative variance was computed to compare how much each method varies from the pooled variance (Uchenna et al., 2012). The correct parameter setting is as shown in Fig. 8 (c), in which the threshold and contrast value are about 50 , the position of spot is in the middle of $k 3$ and $k 4$ regions. This is then the optimal operating mode.

When patients with MND, such as ALS, wave the light source $X(S)$ by swinging their head, the CCD camera converts the optical signal into electronic signal and enters the head gesture interface system in the PC for calculating $H(S)$. Then the calculated result is transmitted to PLC P (S), and the output relay $S(S)$ is controlled by using PLC built-in the control loop. The action $Y(S)$ of household electric appliance is controlled by the relay contacts or the output driver and action of motor or mechanical arm are controlled by using $A / D$ module. The feedback signals are judged by eyes, so that the error detecting program of PC can be used to make feedback signals, in order to reduce the instability of the system. (2) There are software faults and hardware faults in the head gesture interface system. The former one focuses on debugging, and the latter one focuses on random failure, the common cases include crash of PC, virus, and base plate fault. The parallel system can improve the reliability of the system, and prevent software and hardware faults.

Fig. 8 (a) Parameter setting; (b) poor threshold setting; (c) correct parameter setting

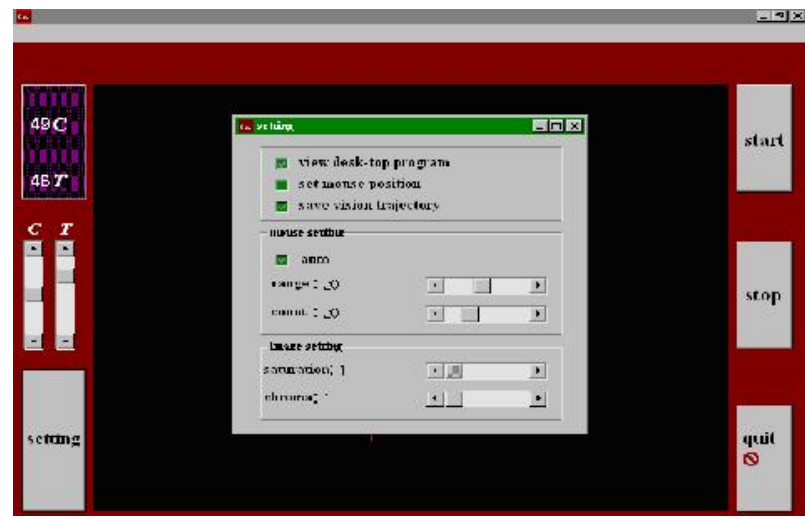

(a)

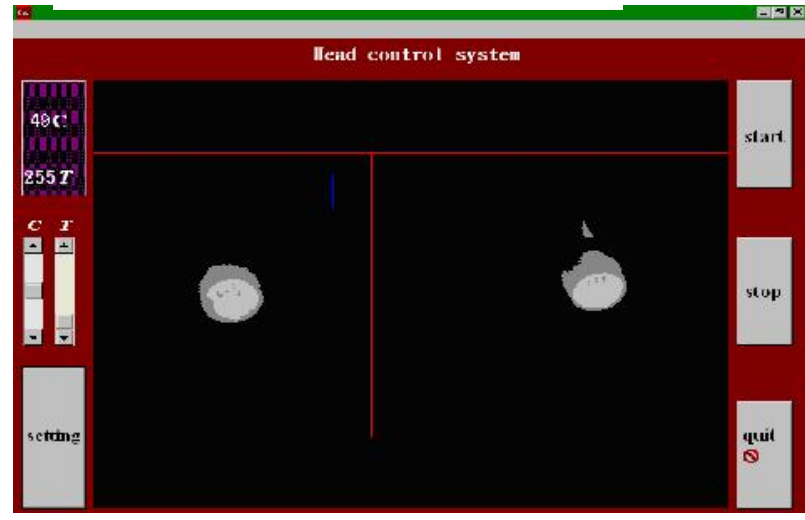

(b)

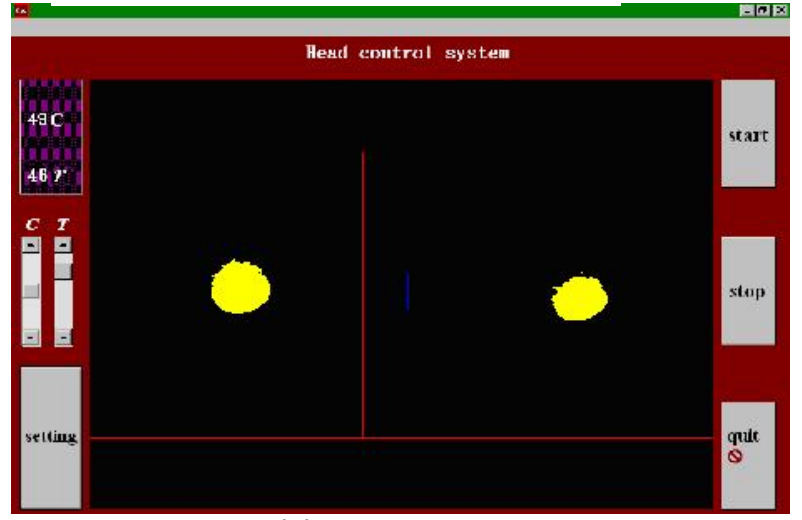

(c)
Research article

CIndian Society for Education and Environment (iSee)
"Head gesture interface" http://www.indjst.org
C-S Lin et al. Indian J.Sci.Technol. 
The parallel operation of low order parallel serial assemblies or programs can reduce the failure rate of system, and it is cheaper than the high order parallel series, including the parallel connection of head gesture interface system program, parallel connection of double monitoring system program, and the parallel connection of double PLC program.

Conclusion

In this paper, we describe a new data processing method which is expected to detect efficiently the position of the head. The system reliability was evaluated by ablebodied subjects for 60 day testing duration. This head controlled system requires patients to wear glasses on which two LED emitters are mounted. The main features of this system are listed as the follows:

1. The previously used computer eyes image capture card is replaced by a new image capture card, and image signals are captured through the image capture card by using SDK provided by the manufacturer, and then exported to PC for calculation.

2. It edits multinational language voice database. Some voice selections are added, such as Thai, Indonesian, Pilipino, Hakka, Taiwanese, etc., so the disabled persons can have convenient communication with their families or nurses.

3. It is designed with the head gesture interface system and PLC image monitoring, in order to develop a userfriendly man-machine interface (MMl). It uses the existing $\mathrm{C}++$ program language to design the program on $\mathrm{PC}$, develops the required $\mathrm{I} / \mathrm{O}$ interface (hardware) and image control menu (software), and carries out on-line monitoring between PC and PLC.

4. It has a complete mechanical-electric integration of head gesture interface system and DCS, and uses the head gesture interface system and PLC image monitoring as SCADA. It also designs PLC program and ladder diagram to complete the wiring and control the household electric appliances, and share data through communication network and home medical control system.

5. It calculates the reliability of the head gesture interface system according to the experimental data, analyzes the causes of failure, and estimates the effective life.

6. The system described in this paper provides an economical and effective method for reliability, and is useful in the field of interface and assistive technology.

\section{Acknowledgement}

This research project was supported by National

Science Council, under grant no. NSC 99-2221-E-035 088 -MY3.

\section{References}

1. Allison RS, Eizenman M and Cheung B (1996) Combined head and eye tracking system for dynamic testing of the vestibular system. IEEE Trans. Biomed. Eng. 43(11), 1073-1082.

2. Betke M, Gips J and Fleming P (2002) The camera mouse: visual tracking of body features to provide computer access for people with severe disabilities IEEE Trans. Neural Sys. \& Rehabilitation Eng. 10(1), 1-10.

3. Barr P, Noble $J$ and Biddle R (2007) Video game values: Human-computer interaction and games. Interacting with Comput. 19, 180-195.

4. Chen YL (2001) Application of tilt sensors in humancomputer mouse interface for people with disabilities. IEEE Trans. Neural Sys. \& Rehabilitation Eng. 9, 289-294.

5. Huang W and Askin RG (2003) Reliability analysis of electronic devices with multiple competing failure modes involving performance aging degradation. Quality \& Reliability Eng. 19, 241-254.

6. Ju JS, Shin Y and Kim EY (2009) Vision based interface system for hands free control of an intelligent wheelchair. J. Neuro Eng. \& Rehabilitation. 33, 1-17.

7. Lee C (1999) Eye and head coordination in reading: roles of head movement and cognitive control. Vision Res. 39, 3761-3768.

8. Lin CS (2002) An eye behavior measuring device for V.R. System. Optics \& Lasers in Eng. 38(6), 333-359.

9. Lin CS, Huan CC, Chan CN, Yeh MS and Chiu CC (2004) The design of a computer game using an eye tracking device for eye's activity rehabilitation. Optics \& Lasers in Eng. 42(1), 91-108.

10. Lin CS, Shi TH, Lin CH, Yeh MS and Shieh HZ (2006a) The measurement of the angle of a user's head in a novel head-tracker device. Measurement. 39(8), 750-757.

11. Lin CS, Ho CW, Chang KC, Hung SS, Shei HJ and Yeh MS (2006b) A novel device for head gesture measurement system in combination with eye-controlled human-machine interface. Optics \& Lasers Eng. 44(6), 597-614.

12. Lin CS, Ho CW, Chan CN, Chau CR, Wu YC and Yeh MS (2007) An eye-tracking and head-control system using movement increment-coordinate method. Optics \& Laser Technol. 39(6), 1218-1225.

13. Lin CS, Chang CC and Chen WL (2008) Design and application of an interactive wheelchair training system, Biomed. Eng: Appl. Basis \& Commun. 20(6), 377-385.

14. Lin CS, Lay YL, Lin CC, Chiu CC and Yeh MS (2009a) Image password lock system by tracing position information of the pupil. Optica Applicata. 39(1), 149-160.

15. Lin CS, Li KC, Chen CT, Chang CC and Chung DS (2009b) Hand gesture recognition in a leg sport system. Biomed. Eng: Appl., Basis \& Commun. 21(2), 97-105.

16. Lin CS, Chen CT, Shei HJ, Lay YL and Chiu CC (2012) Development of a body motion interactive system with a weight voting mechanism and computer vision technology. Optics \& Laser Technol. 44(6), 1934-1941.

17. Lin TS and Chiou SB (1997) The application of Petri nets to failure analysis. Reliability Eng. \& Sys. Safety. 57, 129142.

18. Peraira CMM and Mello RFD (2011) Learning process behavior for fault detection. Int. J. Artificial Intelligence Tools. 20(5), 969-980.

19. Uchenna PO and Nduka EC (2012) Methods of analysing missing values in a regression model. Indian J.Sci. Technol. 5(1), 2013-2016

20. Yoon HS, Soh J and Bae YJ (2001) Hand gesture recognition using combined features of location, angle and velocity. Pattern Recogn. 34, 1491-1501.
Research article

(C)Indian Society for Education and Environment (iSee)
"Head gesture interface"

http://www.indjst.org
C-S Lin et al. Indian J.Sci.Technol. 